\section{KOMPASS}

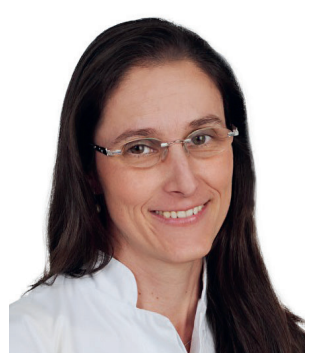

Nóra Szentmáry

Klinik für Augenheilkunde,

Universitätsklinikum des Saarlandes,

Homburg/Saar, Deutschland

Klinik für Augenheilkunde, Semmelweis Universität Budapest, Budapest, Ungarn

\section{Walter Lisch}

Klinik für Augenheilkunde, Johannes Gutenberg Universität Mainz, Mainz, Deutschland

\section{Fabian Fries}

Klinik für Augenheilkunde,

Universitätsklinikum des Saarlandes,

Homburg/Saar, Deutschland

\section{Zoltán Zolt Nagy}

Klinik für Augenheilkunde,

Semmelweis Universität Budapest,

Budapest, Ungarn

\section{Gábor Mikala}

Abteilung für Hämatologie und Stammzelltransplantation,

Zentrales Krankenhaus Süd-Pest, Nationales Institut für Infektiologie und Hämatologie, Budapest, Ungarn

\section{Berthold Seitz}

Klinik für Augenheilkunde,

Universitätsklinikum des Saarlandes,

Homburg/Saar, Deutschland

\title{
Okuläre Signifikanz der monoklonalen Gammopathie
}

Plasmazellerkrankungen umfassen beschwerdefreie, stabile Zustände bis hin zu progressiven Tumoren mit ausgeprägter Symptomatik.

Monoklonale Gammopathie unklarer Signifikanz (MGUS), solitäres Knochenplasmozytom, Morbus Waldenström (Makroglobulinämie) sowie symptomatisches und asymptomatisches Multiples Myelom sind alles unterschiedliche Stadien derselben Erkrankung, die den Nachweis einer monoklonalen Gammopathie gemeinsam haben [1].

Gesunde Plasmazellen produzieren Antikörper, die gegen Infektionen schützen. Diese Antikörper können aus 5 unterschiedlichen schweren Ketten (Immunglobulin-G, Immunglobulin-A, Immunglobulin-M, Immunglobulin-D, Immunglobulin-E) und 2 unterschiedlichen leichten Ketten (Kappa und Lambda) bestehen. Die Anzahl dieser Ketten ist bei MGUS pathologisch verändert. Die Diagnose der monoklonalen Gammopathie wird erst nach Serumproteinelektrophorese und Immunfixation gestellt. Im Rahmen von MGUS ist das sogenannte MProtein als prominente Bande in der Serumproteinelektrophorese nachweisbar [1].

«In der veröffentlichten Literatur wurden insgesamt nur 73 Patienten mit paraproteinämischer Keropathie beschrieben.»

Paraproteinämische Keratopathie (PPK) bei hämatologischen Erkrankungen wurde erstmals Anfang 1900 beschrieben, wobei die erste histologische und immunhistologische Beschreibung aus dem Jahre 1978 von Klintworth stammt. 2016 beschrieben
Lisch et al. 22 Augen (11 Patienten) mit paraproteinämischer Keratopathie als «chamäleonartige Trübungen», während in der veröffentlichten Literatur insgesamt nur 73 Patienten beschrieben wurden $[3,4]$.

Eine PPK findet sich nicht bei allen Patienten mit monoklonaler Gammopathie. In einer Serie von 53 Patienten (106 Augen) mit monoklonaler Gammopathie konnten wir bei nicht mehr als bei 2 Patienten (4 Augen) eine PPK nachweisen. Eine Patientin wies lediglich eine Hornhauttrübung im Sinne einer «monoklonalen Gammopathie mit okulärer Signifikanz» auf, bei dem zweiten Patienten lag die Diagnose eines Multiplen Myeloms vor.

Ob die PPK durch Ablagerung der monoklonalen Proteine in der Hornhaut oder durch Produktion durch Keratozyten (Keratozyten mit Eigenschaften hämatopoetischer Stammzellen) hervorgerufen wird, bleibt noch abzuklären. Dies könnte einen gezielten Therapieansatz der monoklonalen Gammopathie mit okulärer Signifikanz ermöglichen [5].

Bis dahin sollte bei Patienten mit unklaren, bilateralen chamäleonartigen Hornhauttrübungen eine monoklonale Gammopathie durch Serumproteinelektrophorese ausgeschlossen werden, um eine notwendige systemische Therapie dieser hämatologischen Erkrankung ggf. frühzeitig einleiten zu können.

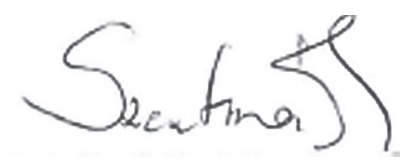

Prof. Dr. Nóra Szentmáry

\section{KARGER}

Fax +497614520714

information@karger.com

www.karger.com

\section{(C) 2019 S. Karger GmbH, Freiburg}

Accessible online at: www.karger.com/kop
Prof. Dr. Nóra Szentmáry

Klinik für Augenheilkunde

Universitätsklinikum des Saarlandes, UKS

Kirrberger Straße 1, Gebäude 22, 66424 Homburg/Saar, Deutschland

nora.szentmary@uks.eu 


\section{References}

1 Dispenzieri A, Kyle R, Merlini G, et al.: International Myeloma Working Group guidelines for serum-free light chain analysis in multiple myeloma and related disorders. Leukemia 2009;23:215-24.

-2 Klintworth GK, Bredehoeft SJ, Reed JW: Analysis of corneal crystalline deposits in multiple myeloma. Am J Ophthalmol 1978;86:303-13.
Lisch W, Saikia P, Pitz S, et al.: Chameleon-like appearance of immunotactoid keratopathy. Cornea 2012;31:55-8.

4 Lisch W, Wasielica-Poslednik J, Kivelä T, et al.: The hematologic definition of monoclonal gammopathy of undetermined significance in relation to paraproteinemic keratopathy (An American Ophthalmological Society Thesis). Trans Am Ophthalmol Soc 2016;114:T7.
5 Németh O, Tapasztó B, Tar S, et al: Corneal deposits in monoclonal gammopathy of undetermined significance. Review of the literature and case report. Orv Hetil 2018;159:1575-83. 